[12] E. KAISER, Microbiologie agricole, p. 506, Paris 1914.

[13] O. BaIL, Bulletin technique des Sciences médicales, t. I, 1925.

[14] F. D’Héret.ue, Bulletin technique des Sciences médicales, t. I, 1925.

[15] R. Bruynogue, Bulletin technique des Sciences médicales, t. I, 1925.

[16] P. Hauduroy, Bulletin technique des Sciences médicales, t. I, 1925.

[17] J. Bondet, Ann. Pasteur, t. XXXIX, p. 718, 1925.

[18] P. Hauduroy, le Bactériophage de d'Hérelle, Paris, Le Franģois, 1925.

[19] H. von Preisz, Die Bakteriophagie, Jéna, Fischer, 1925.

[20] E. Wollman, Ann. Pasteur, t. XXXIX, p. 789, 1925.

[21] V. Brudny, Centralblatt $f$. Bacteriologie, II Abteilung, t. XXII, p. 193, 1909.

\title{
DE LA CONSERVATION ET DE L'ASSAINISSEMENT DU LAIT
}

Par le $D^{r}$ Henri STASSANO

- FIN -

Dans la figure 18 on voit représenté l'un des réfrigérants "Lawrence ", en service dans la Laiterie Centrale de Strasbourg. Ce réfrigérant naturellement peut refroidir aussi bien le lait qui vient directement du pasteurisateur, que le lait provenant de l'un des chambreurs du procédé de la pasteurisation basse.

Nous verrons plus loin, d'après le contrôle bactériologique, quelle est la place que ce système de pasteurisation mérite de prendre. Cependant, ce n'est qu'à la fin de ce mémoire, lorsque nous aurons examiné tous les autres différents aspects de la question et soumis à des contrôles, aussi objectifs, les résultats obtenus en traitant toujours simultanément le même lait cru par les différents procédés de pasteurisation envisagés, qu'il sera possible de tirer une conclusion impartiale.

Au sujet de la stérilisation, en tant que procédé en vase clos, il n'y a pas grand chose à dire. Je tiens seulement à recommander à cette place aux puériculteurs qui, forcément, n'ont pas notre longue habitude du laboratoire, de ne pas se laisser tromper par les affirmations toutes fallacieuses des fabricants de laits stérilisés, au sujet de ces prétendus laits stérilisés à $105^{\circ}$ ou même à $108^{\circ}$ seulement. Même en réduisant le volume des bouteilles de lait dans le triple but d'augmenter l'efficacité du chauffage, de diminuer la coloration et le goût de cuit que le lait prend à l'autoclave, et afin de pouvoir vendre le lait plus cher, sans que le client, peu porté aux calculs, puisse s'en rendre compte, il est indispensable d'atteindre les $112^{\circ}$ pour obtenir la stérilisation du lait.

A l'égard des laits stérilisés, les puériculteurs se laissent également leurrer par l'idée que ces laits au moins ont été chauffés aussitôt après la traite. Eh bien, il suffira de pratiquer dans ces laits le titrage 
des acides aminés pour que l'on ait la preuve que ces laits, comme la grande généralité des laits pasteurisés, ont été chauffés tout aussi bien lorsque une partie de ces acides a fortement diminué à la suite de la multiplication des ferments lactiques. Je reviendrai plus loin sur la signification et la portée de la présence réduite des acides aminés dans les laits ayant à servir pour l'alimentation des enfants.

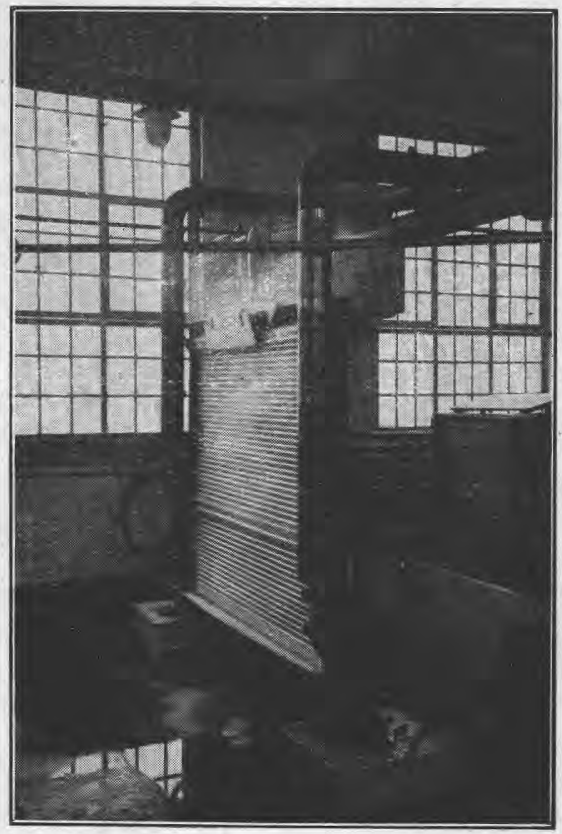

Fig. 18.

Pour obtenir du lait stérilisé à l'autoclave avec moins de coloration et de goût de cuit que d'habitude, on a essayé de raccourcir également la durée du chauffage par l'agitation des bouteilles. A cet effet, Gaulin a construit un stérilisateur à grand travail qui est mobile autour d'un axe horizontal creux par lequel arrive la vapeur; on peut ainsi agiter de temps en temps ou communiquer à l'appareil un mouvement complet de rotation.

Cette idée a été reprise dernièrement par M. GorGerat (1), en remplaçant l'autoclave par un bain-marie renfermant un mélange salin à $112^{\circ}$ muni d'un agitateur. Le lait est préalablement désaéré dans l'intention de supprimer l'oxygène que M. Gorgerat retient être la cause du brunissement du lait pendant le chauffage usuel.

(1) Nouveau procédé de stérilisation du lait en vase clos sans autoclave, Le Lait, p. 394, 1924. 
Cet expédient d'agiter dans le bain-marie les bouteilles pour pouvoir diminuer la durée du chauffage n'est pas nouveau, Il a constitué même la grande invention faite par mon camarade préposé à la fabrieation des vaccins pour pouvoir refuser le procédé de la couche mince que j'avais préconisé bien avant la guerre pour la stérilisation des émulsions microbiennes. En effet, grâce à l'adaptation d'un panier basculant automatiquement, à un vulgaire bain-marie, on parvient à réduire, de trois heures à une heure, la durée de la stérilisation des vaccins, dans de grosses ampoules scellées à la lampe. J'ai pu néanmoins montrer aux personnes et autorités, entre autres au professeur WIDAL, que l'observation pouvait intéresser, que de l'émulsion de blane d'œuf dans de l'eau distillée, enfermée pareillement dans une grosse ampoule identique et soumise pendant une heure à côté des ampoules aux vaccins, à ce mode de chauffage à $58^{\circ}-60^{\circ}$, sortait complètement opaque, alors que la même émulsion, après avoir atteint dans mes appareils en couche mince même la température de $75^{\circ}$, gardait toute sa limpidité.

Je me plus de rappeler ce fait à. M. Gorgerat lorsqu'il vint à la Laiterie Centrale de Strasbourg, pour donner une démonstration de sa méthode. D'avance je lui dis que je m'attendais à quelque petite amélioration, particulièrement dans la coloration, mais à rien de plus que des avantages insignifiants, gagnés au prix de la désastreuse désaération.

Je ne me trompai pas; voici en résumé les résultats obtenus dans l'expérience du 23 janvier 1925 et que la direction de la Laiterie Centrale put contrôler :

Plusieurs bouteilles de lait et quatre bouteilles de crème désaérées, sont chauffées pendant cinq minutes à $100^{\circ}$ et quinze minutes à $115^{\circ}$ avec agitation mécanique.

Constatation : le goût de cuit est' un peu moins accusé que dans les laits stérilisés à l'autoclave, mais très net, la coloration est sensiblement la même que celle du lait eru.

Le lendemain on ouvre une bouteille de crème chauffée et une bouteille semblable simplement désaérée et gardée en chambre frigorifique à $2^{\circ}$. Constatation : la crème simplement désaérée accuse une saveur suiffeuse très forte, pas mangeable, et se bat très mal; la crème chauffée après désaération n'a pas de goût de suif, mais simplement de cuit; ce goût cependant est moins accusé que dans le lait chauffé de la même façon. Cette crème se bat très bien, mais donne lieu à une crème trop épaisse que l'expert pâtissier déclare inutilisable.

Au point de vue bactériologique, la stérilisation était complète.

La stérilisation du lait, par contre, en circulation continue d'après mon procédé, épargne au lait, ainsi qu'à la crème, toute les altérations 
- celles qui sautent aux yeux aussi bien que les altérations qui seules des analyses bio-chimiques' minutieuses peuvent mettre en évidence - que le chauffage à l'autoclave en vase clos provoque fatalement dans le lait. Nous reviendrons plus loin sur le contrôle qui s'impose pour rejeter tout lait qui ne remplit pas les conditions requises d'après des points de repère objectifs et scientifiques.

\section{Valeur comparative de ces différents procédés industriels d'après l'examen bactériologique et les analyses chimico- physiologigues et physiques des laits traités.}

Avant d'entrer dans l'argument, je erois utile d'expliquer(1) en quoi consiste en réalité la pasteurisation du lait en tant qu'action du chauffage sur les microbes de toutes sortes qu'il renferme. On croit généralement que les ferments lactiques qui constituent la plus grande partie de ces microbes sont détruits par la pasteurisation telle qu'on la pratique en laiterie. Il suffit de recueillir et de garder aseptiquement dans des récipients stériles du lait convenablement pasteurisé pour reconnaître que pareille opinion est trop simpliste. Il devient acide et caille comme du lait cru : seul un certain retard le distingue de ce dernier.

Si l'on se borne à l'examen des seules colonies écloses dans les deux ou trois jours qui suivent les ensemencements de l'un et de l'autre lait, on peut partager l'opinion générale exprimée même par quelques spécialistes (2) " que les $99 \%$ des bactéries du lait sont détruites par la pasteurisation ». Mais si, comme il sied, on poursuit l'examen, on voit apparaître sur les milieux nutritifs ensemencés avec du lait pasteurisé, les unes après les autres, toutes les formes microbiennes, à quelques exceptions près, qui poussent précocement entre le premier et le deuxième jour (gélatine, température ordinaire) sur les milieux identiques ensemencés avec du lait cru.

Toutefois, si l'on compare, au moment de son complet développement, vers le huitième jour, la boîte de Pétri ensemencée avec du lait pasteurisé à celle qui correspond au lait cru, au troisième jour de développement (arrêté aussitôt après par le formol), on constate que le nombre des colonies est sensiblement inférieur dans la première. Cette diminution porte évidemment sur les individus moins résistants de chaque espèce. En soumettant, sous couche mince de $1 / 100^{e}$ de millimètre, entre deux plaques métalliques également chauffées, des émulsions de $B$. coli de différents âges, j'ai rencontré, dans des émulsions vieilles de huit jours, des individus supportant

(1) Stassano, Du mode d'action de la chaleur sur les ferments lactiques dans la pasteurisation du lait (C.R. de l'Acad.des Sciences, t. CLXXIX, 1924,p.1438.)

(2) Le Lait, t. I, 1921, p. 106. 
trois degrés et plus au-dessus de la température minima mortelle pour les $B$. coli jeunes de vingt à vingt-quatre heures (1).

Ce qui se passe pour le $B$. coli peut être le fait d'une loi générale. En supprimant les formes bactériennes, à la fois fragiles et plus hâtives, la pasteurisation déterminerait une sorte de sélection : les microbes jeunes succombent, tandis que ceux plus âgés résistent. Je crois avoir déjà la preuve de cette supposition dans le fait que les ferments lactiques qui survivent à la pasteurisation attaquent quelque peu davantage les acides aminés qui se trouvent dans le lait où on les fait fermenter.

Quoiqu'il en soit, mes recherches me permettent désormais d'affirmer qu'une bonne pasteurisation diminue essentiellement la vitalité des microbes du lait, se traduisant par un retard dans leur croissance qui se maintient pendant un certain nombre de générations successives. Chez les ferments lactiques, on peut suivre aisément, par l'analyse acidimétrique, la valeur et la durée de cette modification qui s'efface par degrés progressifs.

Aussi ce ralentissemnent se remarque particulièrement dans le lait pasteurisé d'après le procédé de la couche mince, qui est le procédé dans lequel les microbes sont atteints de la manière la plus uniforme et soutenue.

Voici comment j'ai opéré toujours dans mes essais comparatifs concernant l'action de la chaleur sur les microbes du lait, appliquée selon les trois procédés de pasteurisation dont je me suis occupé depuis trois ans, à savoir : $1^{0}$ celui dit de pasteurisation haute ou

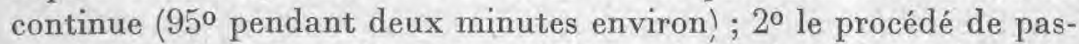
teurisation basse ou discontinue ( $63^{\circ}$ pendant vingt-cinq minutes); $3^{\circ}$ le procédé de la couche mince $\left(75^{\circ}\right.$ et même $70^{\circ}$ seulement pendant huit à dix secondes).

Les trois appareils sont alimentés par le même lait provenant d'une même cuve de 3.000 litres.

Pour la recherche en particulier du degré différent de ralentissement de la vitalité des ferments lactiques à la suite de ces trois systèmes de pasteurisation, j'ai procédé comme il suit; Un tube contenant 15 ce. de bouillon lactosé à $2 \%$ et dont le $\mathrm{P}_{\mathrm{h}}$ mesure 4 à l'échelle des indicateurs colorés, est ensemencé avec une anse de platine du lait cru de la cuve : c'est le tube témoin. Ce degré d'acidité permet aux seuls ferments lactiques de se multiplier. Des tubes contenant le même milieu, sous le même volume, sont ensemencés de la même façon avec une anse de lait de pasteurisation haute, de pasteurisation basse et de lait pasteurisé en couche mince.

Au bout de dix-huit heures d'étuve, l'échantillon témoin accuse un très considérable développement microbien; un peu moins

(1) Stassano, Comples rendus, t. CLV, 1915, p. 820. 
copieux est celui des échantillons de pasteurisation haute et de pasteurisation basse ; par contre, le liquide est à peine troublé dans l'échantillon de pasteurisation en couche mince. Les acidités, exprimées en centimètres cubes de soude $\frac{\mathrm{N}}{4}$, sont respectivement : pour le premier, $17 \mathrm{cc}$; pour le second, $16 \mathrm{cc} .5$; pour le troisième, $16 \mathrm{cc}$, et pour les échantillons en couche mince, 13 cc. 8 , parfois même de 9 à 10 ce. seulement. En prolongeant de vingt-quatre heures le séjour à $37^{\circ}$, le trouble et le dépôt apparaissent dans ces derniers échantillons aussi abondants que dans les autres ; à ce moment, toute différence apparente s'est effacée entre eux. Les mêmes cocco-bacilles y pullulent affectant la disposition de streptocoques et de diplocoques. La production d'acide lactique continue cependant à différer de l'un à l'autre, mais en sens inverse ; voici les valeurs respectives, après trente-six heures d'étuve : 18 cc. $8 ; 19$ cc.; 19 cc. 4 et enfin 20 cc. pour le lait chauffé en couche mince.

Il est d'ailleurs aisé de comprendre à quoi tiennent ces différences et leur ordre renversé. Dans les laits de pasteurisation haute et basse, et encore davantage dans les laits crus, le $B$. mesentericus et le $B$. megatherium se trouvent à l'état de contamination en proportion moins exiguë que dans le lait pasteurisé en couche mince.

Déjà dans l'expérience précédente, lorsqu'on suivait le développement de l'acidité au delà de vingt-quatre heures sur les tubes de bouillon lactosé acide $\left(6 \mathrm{P}_{\mathrm{h}}\right)$, on pouvait remarquer à l'avantage de l'un des trois procédés de pasteurisation sur lesquels portait l'expérience une plus forte acidité. Ce résultat exprimait nettement une plus grande pureté microbienne dans le milieu en question, lequel correspondait au lait de pasteurisation en couche mince.

Par contre, en tenant compte des acidités de moins en moins accusées que l'on décelait dans les autres tubes, on était amené à conclure $: 1^{\circ}$ que dans le tube provenant du lait cru témoin, l'impureté microbienne était la plus forte; $2^{\circ}$ que dans le tube provenant du lait de pasteurisation haute, cette même impureté était un peu moins prononcée; $3^{\circ}$ que, dans le dernier tube, celui provenant du lait de pasteurisation basse, le degré de l'impureté était encore moindre, se plaçant par conséquent entre le degré d'impureté tirant origine du lait de pasteurisation haute et celui tirant origine du lait de pasteurisation en couche mince.

Cette expérience préludait donc au classement par ordre d'efficacité du chauffage sur les microbes du lait qui résulte, on ne peut plus clairement et sûrement, de la simple inspection des résultats obtenus dans une autre de mes nombreuses expériences comparatives sur ce sujet et consignés dans les neuf reproductions photographiques suivantes (fig. 19 à 26 bis). Les chiffres inscrits au-dessus de chacune 
de ces figures, - indiquant la valeur de l'acidité lactique et de l'acidité aminée, présentées après un séjour de dix-huit heures à l'étuve par le même lait dont provient l'ensemencement, dans le milieu nutritif correspondant à la même figure, - renforcent la portée de la simple démonstration visuelle.

Dans l'espèce, il s'agit de neuf boîtes de Bö̈z, en forme de cuvettes, concaves, destinées spécialement pour les hémocultures anárobies en milieu solide. Chaque boîte comporte deux cuvettes de $24 \mathrm{~cm}$. de diamètre, s'emboîtant l'une dans l'autre, ménageant entre elles une cavité d'environ $130 \mathrm{cc}$. dont le volume est réglé par trois nervures disposées en rayon et faisant saillie sur la face coneave de la cuvette inférieure. L'intervalle qui sépare les deux cuvettes est de $3 \mathrm{~mm}$. 5 au centre et s'atténue vers la périphérie. Le milieu de culture que j'ai employé était constitué de gélose à $2 \%$, lactosée à $2 \%$ et répartie en ballons à raison de $110 \mathrm{ec}$.

A pareil volume constant de gélose, redissoute et entretenue liquide au moment de l'ensemencement à la température de $40^{\circ}$ à $45^{\circ}$, ont été mélangés $10 \mathrm{ec}$ de bouillon renfermant $1 / 1000$ de centimètre cube d'un des différents laits à l'étude. Le tout a été ensuite coulé entre les deux cuvettes. Une fois la gélose refroidie - on en facilite Je prompt refroidissement en appliquant contre de l'eau froide la cuvette inférieure - on coule tout autour du cerele de gélose solidifiée un mélange de paraffine et de vaseline qui en se solidifiant, à son tour, protège le mílieu nutritif, qu'il encercle, contre toute eontamination extérieure.

Dans ce milieu très favorable au développement des germes anaérobies, se développent tout aussi bien les anaérobies facultatifs, tels que les staphylocoques, les streptocoques, les B. typhiques. paratyphiques, coli, proteus, etc.

J'ai pensé que ce milieu pouvait convenir également pour les ensemencements des microbes du lait, dans l'espoir d'y voir apparaître, à leur tour, les anaérobies stricts dont la présence ne peut peut être révélée dans les ensemencements en surface et à l'air.

Les ensemencements en gélatine dans les boîtes de PÉTri, dont je me sers habituellement, se prêtent mieux pour le dénombrement des colonies, la couehe étant plus mince et homogène; de plus les boîtes de PÉTri conviennent particulièrement dans l'étude des laits pasteurisés, car le développement se faisant à la température ordinaire on voit apparaitre les colonies des ferments lactiques les unes après les autres, parfois avec des intervalles de deux et même trois jours (dans le cas par exemple du lait pasteurisé en couche mince) selon le degré de dureté de l'épreuve thermique subie par chaque microbe.

Les nouvelles boîtes de Bö̈z offrent par contre d'autres avantages, 




FIG, r9. - Lait cru témoin ( 36 heures de séjour à létuve). Mème lait après 18 heures de séjour à l'étuve : Acidité lactique ; 39 cc. - Acidité aminée : 32,6 cc.

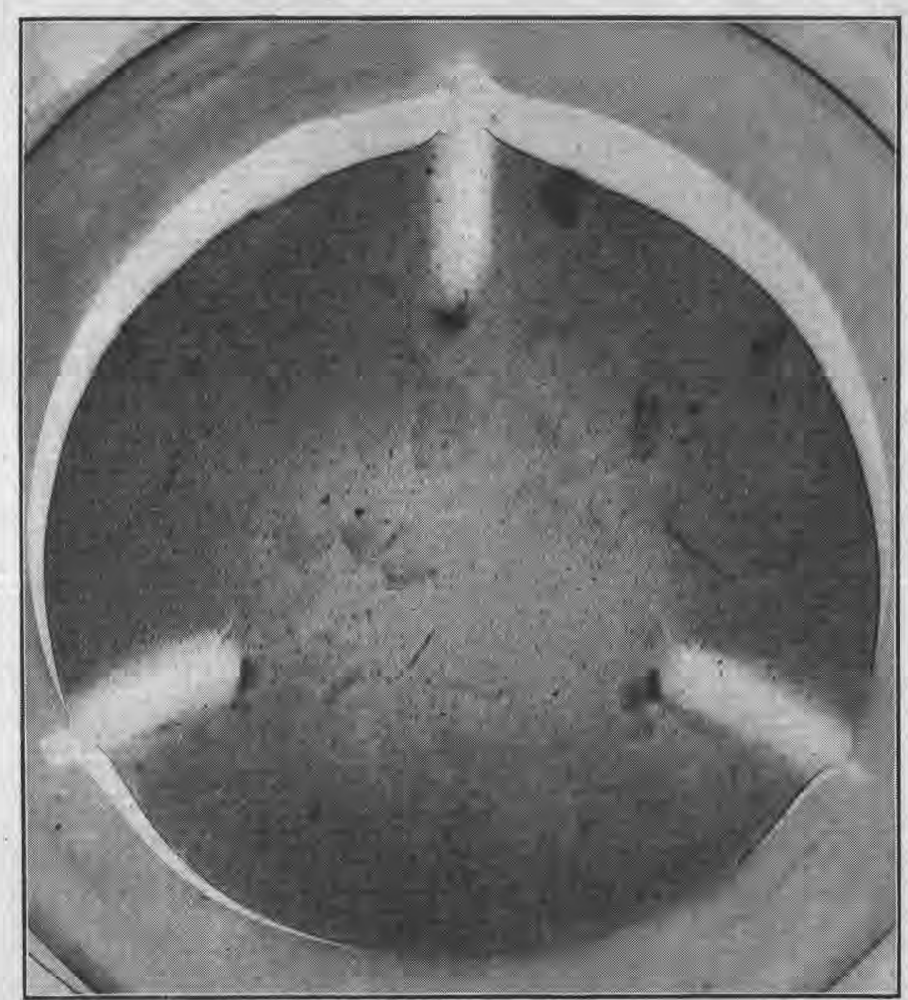

FIG. 20. - Lait cru témoin (24 heures de séjour ả l'étuve). Même lait après 18 heures de séjour à l'étuve : Acidité lactique : $39 \mathrm{cc}$. - Acidịté aminée : $32,6 \mathrm{cc}$. 


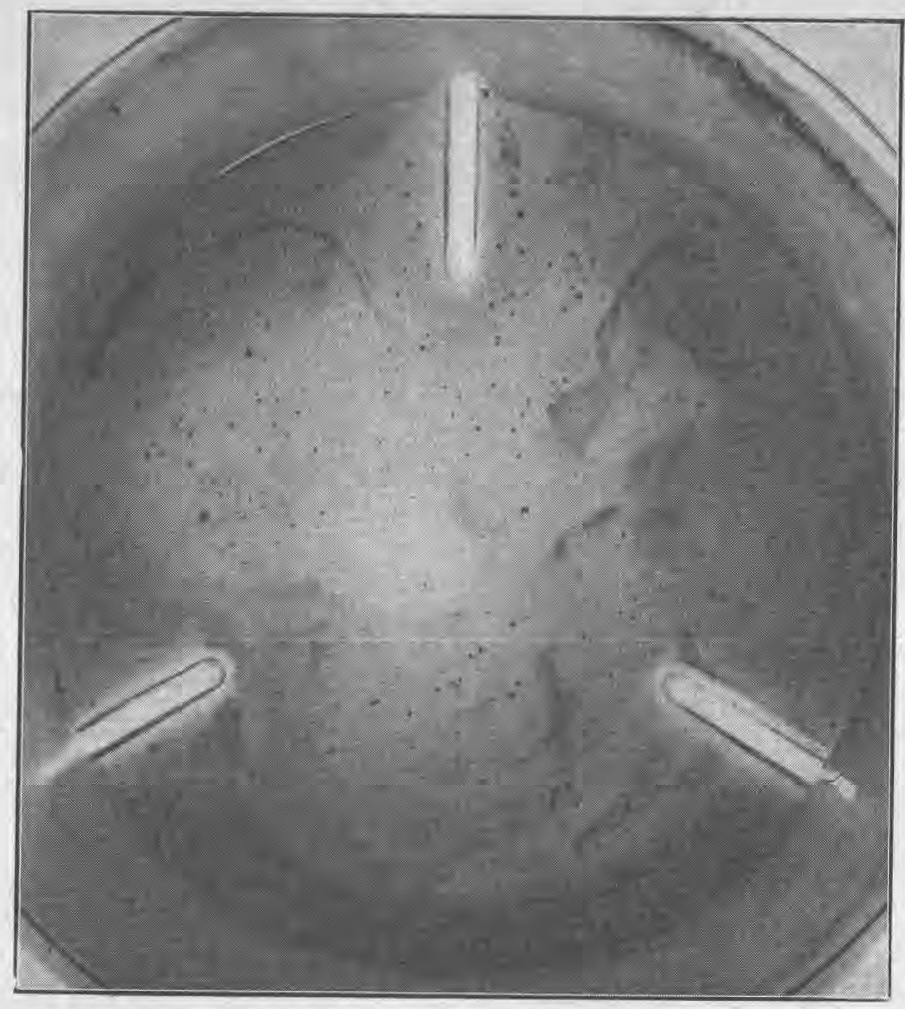

Fig.21. - Lait de pasteurisation haule $85^{\circ}$ ( 36 heures de séjour à l'étuve) Même lait après 18 heures de séjour à l'étuve : Acidité lactique : $36,6 \mathrm{cc}$. - Acidité aminée : $31 \mathrm{cc}$.

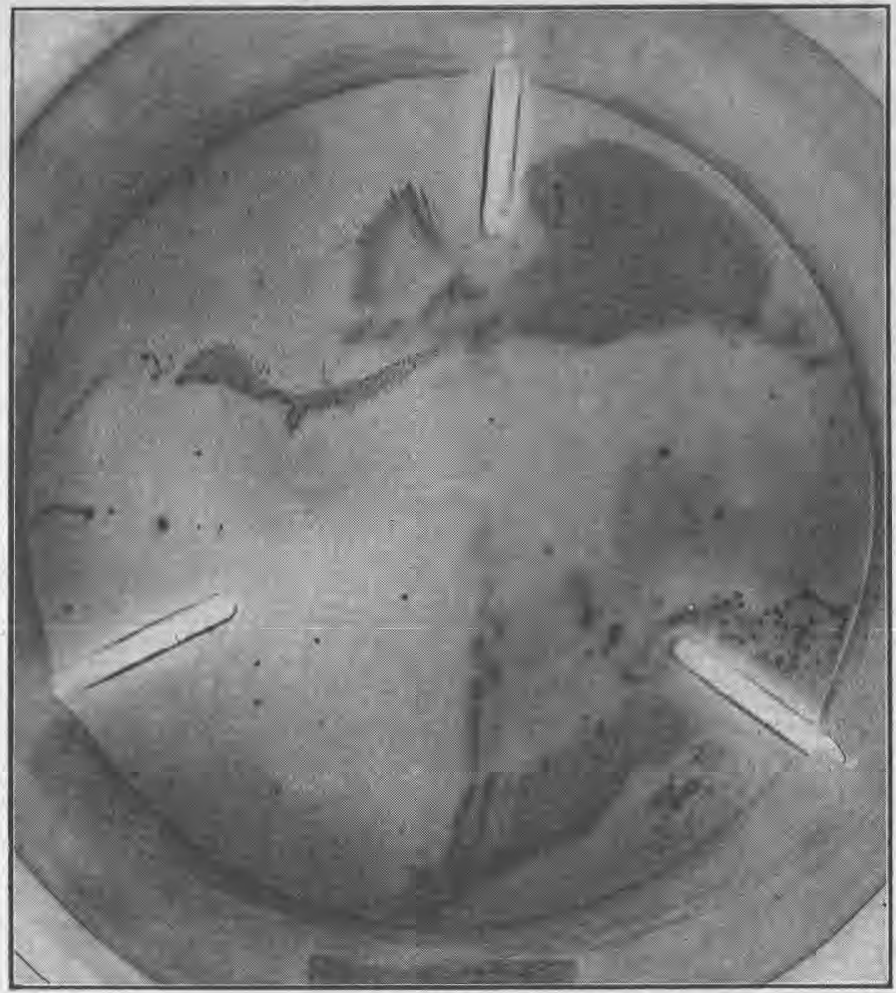

Fig. 22. - Lait de pasteurisation haute $90^{\circ}$ ( 36 heures de séjour à l'étuve). Même lait après 18 heures de séjour à l'étuve : Acidité lactique : $33,6 \mathrm{cc}$. - Acidité aminée : $32 \mathrm{cc}$. 
celui particulièrement de permettre l'éclosion des anaérobies à côté de toutes les autres espèces de microbes.

Pourtant dans les essais que j'ai fait jusqu'ici il s'est produit un résultat bien inattendu avec les ensemencements des laits pasteurisés. Je vais en dire un mot avant de revenir à l'argument qui est le classement des différents procédés de pasteurisation d'après leur valeur microbicide ou simplement retardatrice.

Les anaérobies stricts étant des germes sporulés, ne peuvent pas certainement être supprimés par la pasteurisation quel qu'en soit le procédé employé. Or, jusqu'ici, je n'ai eu de développement de gaz assez important pour faire éclater la couche de gélose comme l'on voit sur la figure 1 que dans les ensemencements des laits crus. Que se passe-t-il dans les ensemencements pratiqués dans les milieux dont il s'agit avec du lait pasteurisé ? L'explication la plus vraisemblable, mais qui demande à être soumise à l'expérience, est que dans

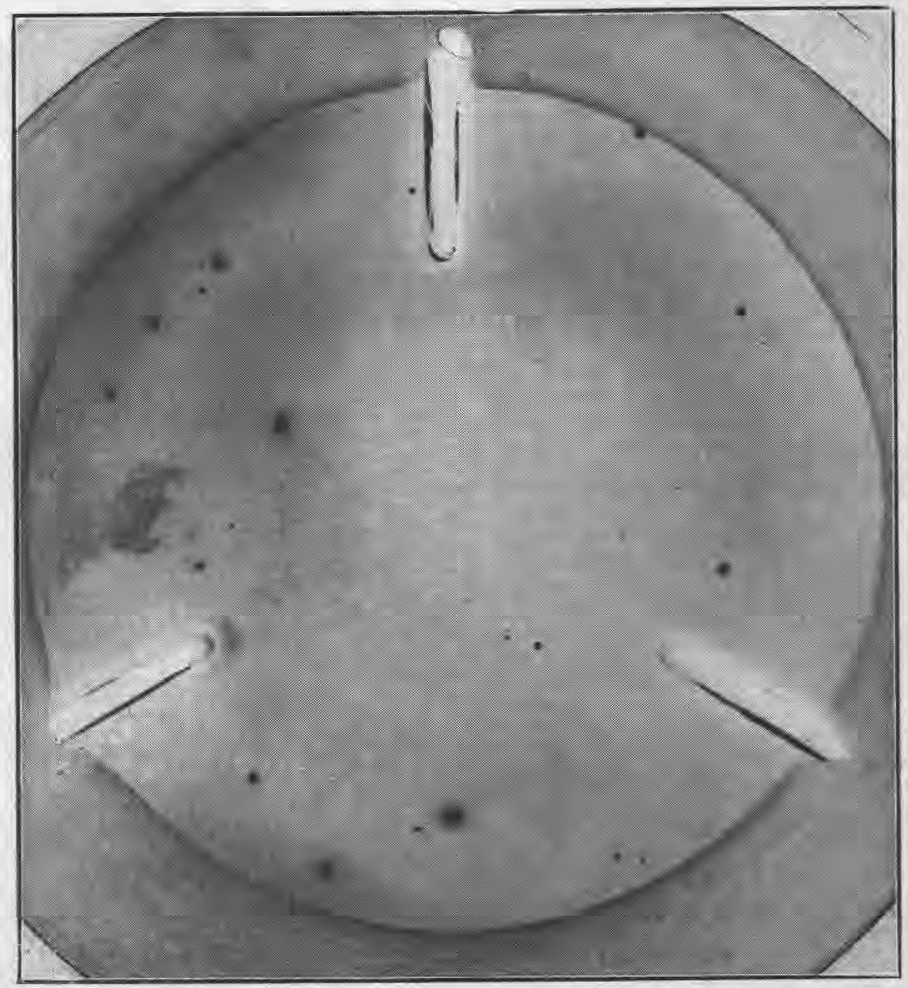

Fig. 23, - Lait de pasteurisation haute $9^{\circ}$ ( 36 heures de séjour à l'étuve). Mème lait après 18 heures de séjour à l'étuve : Acidité lactique : $30,2 \mathrm{cc}$. - Acidité aminée : $38 \mathrm{cc}$. 


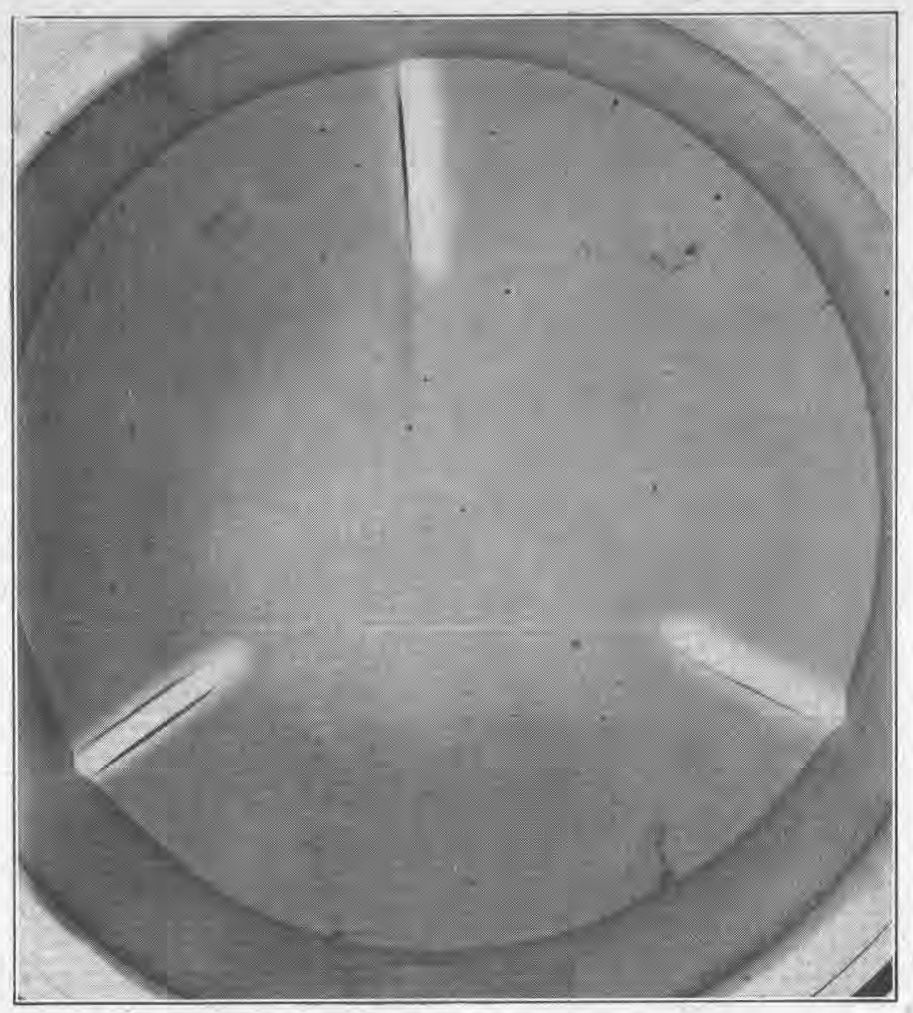

FIG. 24. - Lait de pasteurisation en couche mince $7^{\circ}$ (36 heures * de séjour à l'étuve)

Même lait après 18 heures de séjour à l'étuve : Acidité lactique : ro,6 cc, - Acidité aminée. $4 \mathrm{r} .8 \mathrm{cc}$.

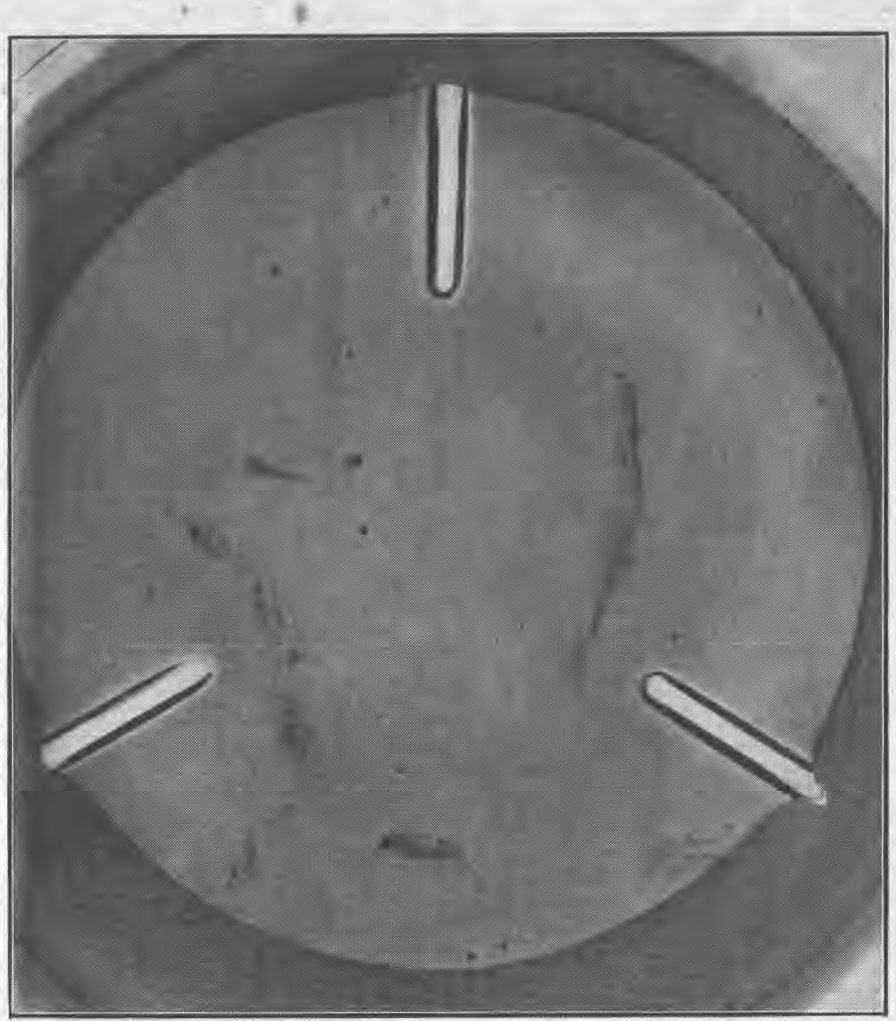

FiG. 25. - Lait de pasteurisation en couche mince $75^{\circ}$ (4 jours de sêjour à l'étuve).

Même lait après $\mathrm{t} 8$ heures de séjour à l'étuve : Acidité lactique : $10,6 \mathrm{cc}$. - Acidité aminée : $41,8 \mathrm{cc}$. 


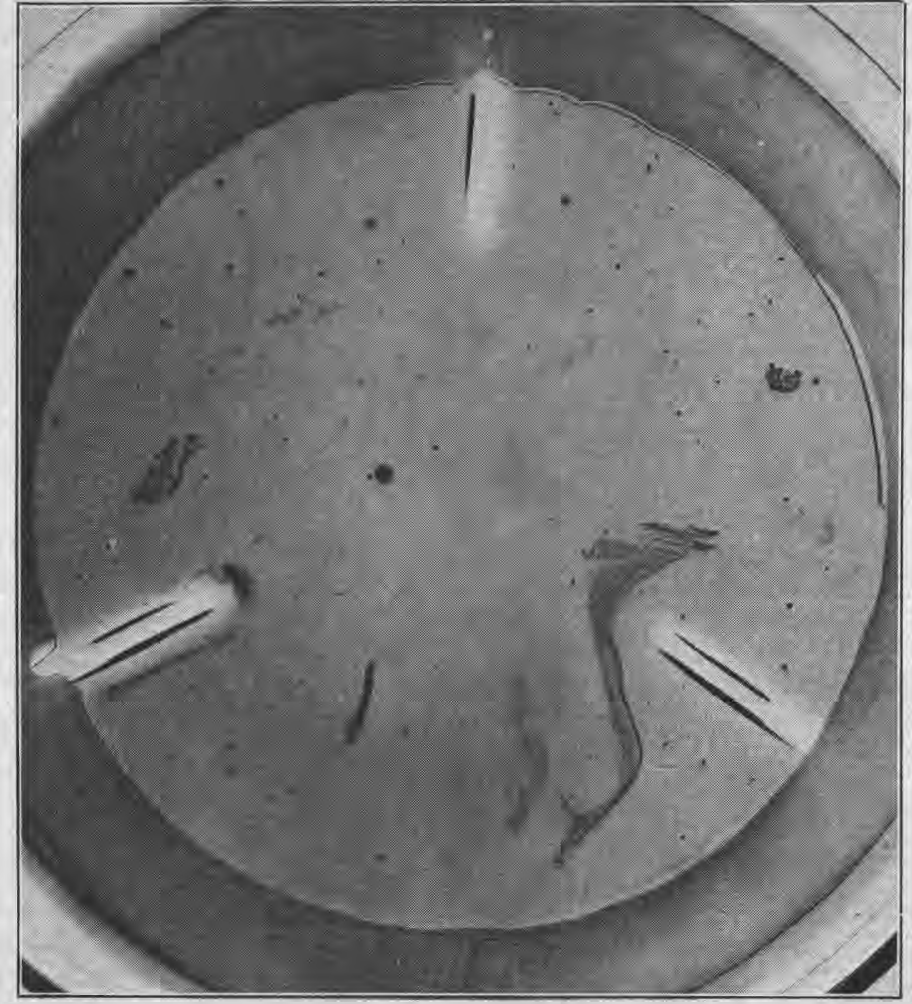

FIG. 26. - Lait de pasteurisation basse $63^{\circ}$ ( 36 heures de séjour á l'étuve). Mème lait après 28 heures de séjour à l'étuve : Acidité lactique : 15,2 cc. - Acidité aminée : 37 cc.

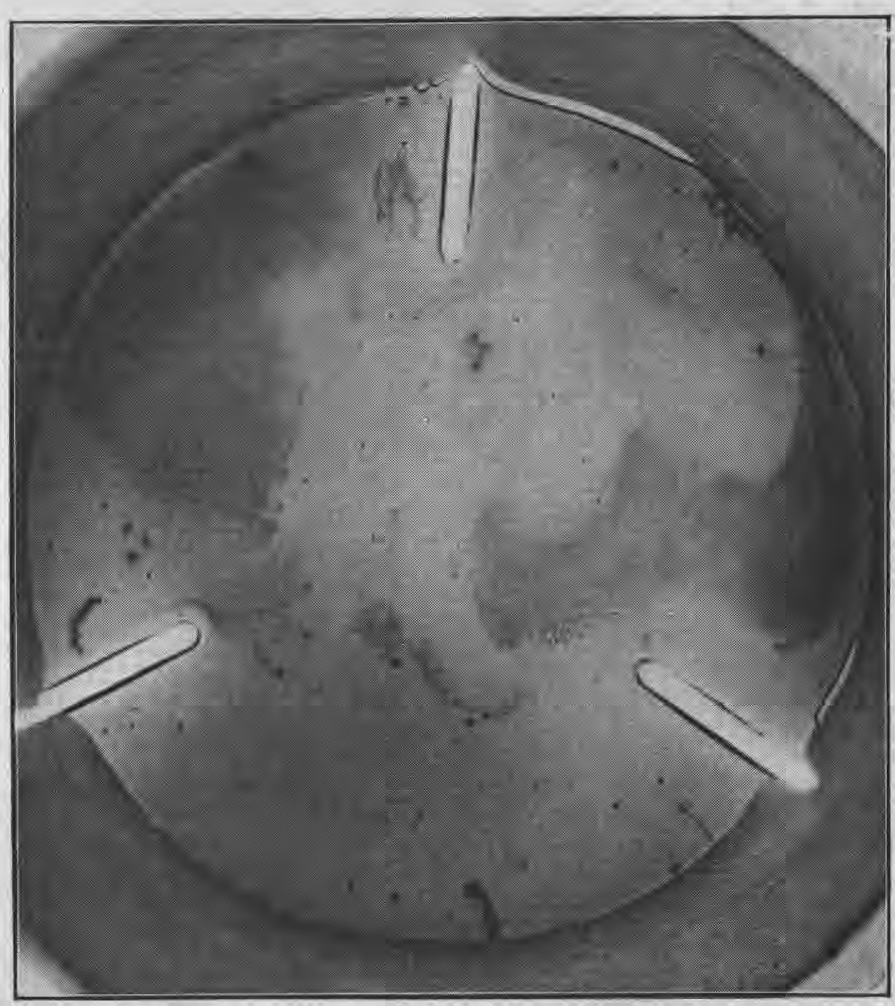

FIG, 26 bis. - Lait de pasteurisation basse $63^{\circ}$ ( 36 heures de séjour à l'étuve). Même lail aprés 18 heures de séjour à l'étuve : Acidité lactique : $15,2 \mathrm{cc}$. - Acidité aminée : $37 \mathrm{cc}$. 
la pasteurisation viendraient à être tués ou fortement ralentis les microbes avec lesquels les germes strictement anaérobies vivent en symbiose et dont dépendrait leur développement.

Revenons à présent au elassement dont les jalons sont représentés par ces neuf figures. Il convient avant tout de comparer la figure 20 avec la figure 21 pour pouvoir porter un jugement assez probant sur la faible valeur de la pasteurisation haute telle qu'elle est pratiquée généralement, e'est-à-dire en portant le lait dans le pasteurisateur usuel à $85^{\circ}$ et en le refroidissant tout de suite après.

La figure 20 est la plaque ensemencée avec 1/1000 de centimètre eube du lait cru témoin après un séjour de vingt-quatre heures à l'étuve ; la figure 24 représente par contre la plaque ensemencée avec la même quantité de lait, mais de lait de pasteurisation haute à $85^{\circ}$, après un séjour plus long à l'étuve, trente-six heures au lieu de vingtquatre. Sur l'une et sur l'autre plaque, on apercoit nettement de très nombreuses colonies, sur la seconde cependant environ le tiers des colonies essaimées sur la première.

Parallèlement à cette baisse dans le taux primitif en microbes du lait $\mathrm{cru}$, à la suite de la pasteurisation haute à $85^{\circ}$, il s'eśt produit dans l'échantillon correspondant à ce même lait exposé pendant dix-huit heures à l'étuve ine formation moindre d'acide lactique que dans le lait témoin et vice-versa la valeur de l'acidité aminée a diminué davantage dans ee dernier que dans le lait pasteurisé en question. C'est ce que témoignent les chiffres inserits en dessous des deux figures.

Poussant plus loin l'examen sur le lait de pasteurisation haute, la plaque ensemencée dans les mêmes conditions avec le lait chauffé à $90^{\circ}$, figure 22 , accuse encore beaucoup moins de colonies et dans l'échantillon de lait correspondant on constate les acidités suivantes, après la même durée de séjour à l'étuve : 33,6 , acidité lactique ; 32 , acidité aminée; la plaque ensemencée pareillement avec du lait de pasteurisation haute, mais à la température supérieure de $95^{\circ}$ (figure 23), accuse encore une plus forte baisse microbienne, quelques colonies à peine y figurent, qui se montrent par contre très développées. Elles ont continué de grandir quelque temps même en dehors de l'étuve à la température ordinaire, malgré l'exposition au jour. Respectivement les acidités présentées après dix-huit heures d'étuve par l'échantillon de lait correspondant, sont 30,2 : lactique et 38 : aminée.

Pour pouvoir comprendre à quoi tient pareille chute dans le nombre de microbes existants dans le lait eru et qui survivaient encore après la pasteurisation à $85^{\circ}$, lorsqu'on a chauffé le même lait par le même procédé, mais en lui faisant atteindre les températures plus élevées de $90^{\circ}$ d'abord et ensuite $95^{\circ}$, et pour pouvoir saisir toute la partie 
démonstrative de cette chute et dans les conditions qui l'accompagnent, il faut fixer un instant le regard sur la figure 24, qui représente la plaque qui correspond à l'ensemencement opéré dans les mêmes conditions et proportions, mais avec du lait pasteurisé en couche mince.

Ici encore très peu de colonies, un peu plus que sur la plaque de pasteurisation haute à $95^{\circ}$, seulement dans le cas du lait de pasteurisation en couche mince, les colonies sont très petites, quelquesunes même à peine visibles, alors que les colonies de la plaque au lait de pasteurisation haute à $95^{\circ}$ sont énormes en comparaison des premières. Parallèlement, voilà les chiffres correspondant des acidités respectives : pasteurisation en couche mince : 10,6, lactique et 41,8 , aminée; pasteurisation haute à $95^{\circ}: 30,2$, lactique et 38 , a minée.

La conclusion qui se dégage nettement de ces deux ordres de comparaison, visuelle et numérique, est la suivante; dans le lait de pasteurisation haute à $95^{\circ}$, très peu de microbes, dans les espèces de ferments lactiques, ont échappé à l'action meurtrière de ce mode de pasteurisation, les quelques rescapés cependant n'accusent pas la moindre trace d'affaiblissement; une fois remis dans un milieu nourricier favorable, ils s'y développent aisément mieux que jamais, fabricant beaucoup d'acide lactique et, corrélativement, épuisant davantage la réserve en acides aminés dont est pourvu le lait à l'origine; au contraire, les microbes survécus à l'action uniforme du chauffage sur les bactéries qui caractérise le procédé de la couche mince, se montrent tous très ralentis dans leur vitalité. D'où le faible développement des colonies que chacun d'eux forment sur la plaque correspondante, la faible acidité lactique : 10,6 qu'accuse l'échantillon de lait correspondant après les dix-huit heures de séjour à l'étuve et la forte acidité aminée que ce même lait accuse à son tour : 41,8, très rapproché du taux normal du lait eru en acides aminés.

Sur la plaçue figure 25 , correspondant au même lait de pasteurisation en couche mince, mais qui est restée à l'étuve pendant quatre jours, on remarque quelques colonies de plus, apparues dans la suite; ce sont surtout des colonies écloses à la surface de la gélose formant sous la plaq̨ue de verre de larges taches rappelant les nébuleuses dans un ciel étoilé ; mais ce développement, numériquement parlant, est bien loin d'égaler celui qui se produit lorsqu'on suit pendant plusieurs jours de suite, jusqu'à huit, en boîtes de PÉtri, sur gélatine, l'apparition retardée des ferments lactiques provenant d'une opération de pasteurisation en couche mince. J'ai déjà signalé plus haut ce fait.

Enfin, les plaques figure 26 et 26 bis, qui correspondent au lait de paseurisation basse, montre que ce procédé apporte certainement un avantage à l'égard de l'action du chauffage sur les bactéries du lait 
vis-à-vis du procédé de la pasteurisation haute, notamment lorsque dans la pratique de ce procédé on n'atteint pas les températures élevées de $90^{\circ}$ et $95^{\circ}$.

D'ailleurs, la valeur des deux acidités respectives de ce lait, après la même épreuve de dix-huit heures à l'étuve, 15,2, lactique et 37, aminée, démontre que dans ce lait les ferments lactiques ont été d'une façon bien plus homogène atteints par le chauffage bien qu'à un degré considérablement inférieur, comme uniformité et intensité d'effet que dans la pasteurisation en couche mince.

La survie de ces quelques colonies de ferments lactiques et en si fleurissant état de vie dans les laits de pasteurisation haute, chauffés aux températures extrêmes, exceptionnelles, de $90^{\circ}$ et même de $95^{\circ}$, a, en plus de ce que je viens d'exposer au sujet du classement des différents procédés, une valeur toute particulière. Car elle apporte la preuve la plus éclatante de cette irrégularité, de cette incertitude d'action du chauffage pratiquê par ce procédé, dont depuis Soxhlet tous les puériciculteurs se doutent et se méfient et pour remédier à laquelle on a préconisé l'expédient de garder chaud le lait quelque temps après la sortie du pasteurisateur avant de le refroidir.

S'il y a, en effet, des ferments lactiques qui échappent si bien à l'atteinte de ce mode de pasteurisation, bien d'autres microbes, parmi lesquels les germes pathogènes, peuvent y échapper : voilà la preuve qu'apporte l'expérience dont on trouve les éléments, j'ajouterai les pièces à conviction, dans les figures 19 à 26 bis.

L'épreuve par contre la plus uniformément dure déterminée par ce mode de procédé de pasteurisation haute, porte malheureusement, mais d'une facon indéniable, sur les constituants albuminoïdes, gazeux et minéraux du lait.

Cependant, cet ordre d'altération n'est pas exclusif de la pasteurisation haute; par les autres procédés de chauffage, ces mêmes altérations se produisent encore, mais à un degré moindre; nous entrons ainsi dans la seconde partie de chapitre, concernant particulièrement les caractères chimico-physiologiques et physiques que présentent les laits traités par les différents procédés thermiques dont il a été question jusqu'iei.

Il y a, en premier lieu, des altérations qu'il n'est pas possible d'évaluer par des mesures absolues, mais uniquement par des comparaisons. Tel est notamment le cas de la faculté qu'a le lait frais de cailler par addition de présure ou de présenter les réactions des peroxydases et des réductases : propriétés que les différents procédés en question diminuent à des degrés différents.

Vis-à-vis de ces propriétés, il faut donc opérer chaque fois comparativement, en prenant comme termes de comparaison le lait cru témoin et utilisant des réactifs dont on sait d'avance le mode d'action 
et les proportions les plus avantageuses pour la production des réactions. Opérant de la sorte, on trouve d'une façon constante que le lait de pasteurisation en couche mince se comporte à peu près comme le lait cru correspondant; le lait de pasteurisation basse se place après; enfin vient le lait de pasteurisation haute, caractérisé par l'absence de la réaction de la peroxydase, tout au moins par le procédé de Sтовсн, et par la difficulté à cailler par la présure.

Pour pouvoir juger d'une facon moins imprécise du degré d. conservation des constituants du lait, particulièrement des plus fragiles, à la suite des différants procédés de pasteurisation et de chauffage en"général du lait, j’ai entrepris deux séries différentes de déterminations : la première porte sur les principales constantes physico-chimiques du lait cru et du même lait après avoir subi les trois procédés de pasteurisation objets de mes essais comparatifs ; la seconde concernant les variations des équilibres chimiques du lait provoquées par l'action de la pasteurisation selon ces mêmes procédés et subsidiairement par le vide.

Dans la première (1), j'ai eu comme collaboratrice MHe G. Acнard et le travail a été exécuté dans l'Institut de chimie-physique et biologique de la Faculté de Médecine de Strasbourg.

Nous avons examiné quelques constantes physico-chimiques du lait, en viue d'apprécier les modifications qu'y apporte le chauffage, d'après différents procédés de pasteurisation.

Les procédés de pasteurisation que nous avons suivis à cet égard sont, répétons le :

$1^{0}$ Le procédé de pasteurisation haute ou continue ; chauffage à $95^{\circ}$ pendant deux ou trois minutes;

$2^{\circ}$ Le procédé de pasteurisation basse ou discontinue : chauffage à $63^{\circ}$ pendant 25 à 30 minutes ;

$3^{\circ}$ La pasteurisation continue en couches minees, huit à dix secondes de chauffage à $75^{\circ}$.

Pour chaque expérience, les divers appareils de pasteurisation ont été alimentés simultanément par le même lait eru, un témoin a été observé cru. Ainsi les résultats sont comparables. Ces précautions sont indispensables, car, d'après les travaux de divers auteurs, les constantes physico-chimiques du lait varient beaucoup d'un échantillon à l'autre.

Enfin, les échantillons soumis à expérience sont recueillis dans des récipients stériles et conservés en glacière jusqu'au moment de leur utilisation.

Dans ces conditions, les résultats suivants ont été observés :

Le $\mathrm{P}_{\mathrm{h}}$ des divers échantillons a été mesuré par la méthode des

(1) C. R. de la Soc. de Biologie, t. XCIII, p. 708, 1925. 
indicateurs colorés et n'a pas montré de différence entre le lait cru et le lait pasteurisé par les divers procédés $\left(\mathrm{P}_{\mathrm{h}}\right.$ voisin de $\left.6,6-6,7\right)$.

$\mathrm{Si}$ le lait demeure quelques heures à la température ordinaire du laboratoire, le $\mathrm{P}_{\mathrm{h}}$ varie de façon très appréciable par suite de la fermentation lactique. Dans un tel échantillon, le $\cdot P_{h} s^{\prime}$ est montré. aux indicateurs, compris entre 5,1 et 5,6 . Une récente série d'expériences a été effectuée, par la méthode électrométrique, à l'aide de l'électrode Vlès et Vellinger. Quelques faibles différences ont été observées :

Procédé de pasteurisation

$\mathrm{Ph}$ 一

Couche mince

Pasteurisation haute

Pasteurisation basse
Acidité à la phénolphtaléine (ce. $\mathrm{NaOH} \frac{\mathrm{N}}{5}$ à 100 cc. et de lait pour virage an rouge de la phénolphtaléine).

Ces résultats concordent avec les observations récentes de Cosmovicr (1) qui a montré que l'acidité ionique augmente par le chauffage; de même ici, c'est le lait le moins chauffé (pasteurisation, couche mince) qui a le $\mathrm{P}_{\mathrm{h}}$ le plus élevé.

Conductivité. - La conductivité a été mesurée par la méthode de Kohlrausch (Ohmmètre de Chauvin et Arnoux) et n'a pas montré de différences entre le lait cru et les divers laits pasteurisés.

$\mathrm{A}=0,00375 \mathrm{ohms}$ par centimètre cube à $16^{\circ}$ (ou bien $=266 \mathrm{ohms}$ à $\left.16^{\circ}\right)$.

Lesage et Dongier (2) ont trouvé, en 1912, une résistivité variant entre 235 et 265 ohms par centimètre cube à $16^{\circ}$. Nos résultats concordent donc avec les leurs.

Abaissemient cryoscopique. - Les mesures effectuées par la méthode de Raoult-Beckmann (chiffre brut sans correction due à la température du réfrigérant) ont montré des variations de $\Delta$ très faibles et incertaines.

Donnons, à titre d'indication, deux séries d'expériences :

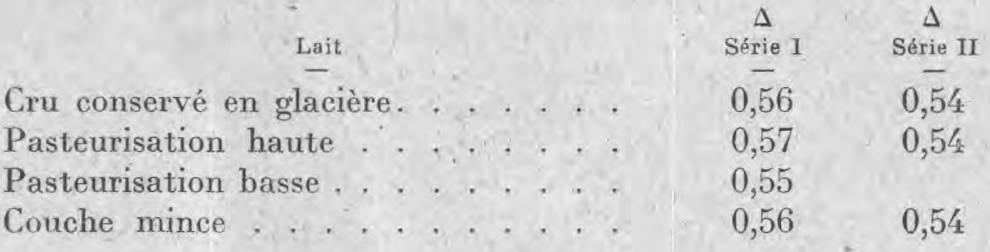

Cependant, l'observation journalière a montré que lorsqu'ori conserve du lait en chambre frigorifique à $-2^{\circ}$, on trouve le lait_cru

(1) Cosmovicr, Bulletin de la Société de Chimie biologique, VII, 2, 1925.

(2) Lesage et Dongier, C. R. Ac. Sc., 1902, 134. 
congelé, tandis que les laits pasteurisés sont encore liquides. La surfusion cesse done plus vite dans le cas du lait cru. D'autre part Bonnema (1) a trouvé que le chauffage élève le point de congélation du lait, ce qui est en désaccord avec nos résultats. Au point de vue valeurs absolues des mesures, nos résultats concordent avee ceux de Winter (2), de Barthel (3), de Pemmington (4), de Porcher et Chevaluer (5), d'après ces derniers auteurs, $\Delta$ oscille autour de 0,555 .

Densité. - Les densités ont été déterminées par la balance de Монг.

\begin{tabular}{ccccc}
\multicolumn{2}{c}{ Exemple : } & & \\
Série & Lait cru & Pasteurisation & Pasteurisation & Couche mince \\
$\overline{1}$ & $1, \overline{0} 2$ & $1, \overline{0} 3^{\prime}$ & 1,031 & 1,032 \\
2 & 1,030 & 1,032 & 1,032 & 1,031 \\
3 & 1,032 & 1,032 & 1,032 & 1,032
\end{tabular}

En somme variations faibles et de sens incertain.

Viscosité. - Les viscosités (relatives à l'eau) ont été déterminées au viscosimètre d'Ostwald. Donnons un exemple :

$\begin{array}{cccc}\text { Lait cru } & \text { Pasteurisation basse } & \text { Pasteurisation haute } & \text { Couche mince } \\ -\overline{1}, 69 & 1, \overline{74} & 1,76 & - \\ \text { n } & & 1,72\end{array}$

Kobler a donné des valeurs de viscosité variant de 1,99 à 2,06. Nos valeurs varient (pour divers échantillons de lait cru) entre 1,69 et 1,83 et entre 1,74 et 2,15 pour des laits diversement pasteurisés.

Tension superficielle. - Mesurée au tonomètre Kopaczewski. Pas de variations appréciables entre les divers échantillons. Valeur obtenue : 48 dynes à $20^{\circ}$.

Conclusion. - Seule la viscosité et le $\mathrm{P}_{\mathrm{h}}$ montrent dans le cas du lait cru et du lait pasteurisé des différences assez appréciables ; dans ces conditions, e'est le lait pasteurisé par la méthode de la couche mince qui se rapproche le plus du lait normal.

Les variations des autres constantes physico-chimiques sont en première analyse, faibles et de sens incertain.

(1) Bonnema, Pharmac. Week. 43, 1906; Milch. Zentralbl. 2, 1906.

(2) Winter, C. R. Ac. Sc., 121, 1895.

(3) Bлвтнец, Revue générale du lait, 4, 1908.

(4) Pemmington, Hopburn, John, Witmer, Dafford, Bundle, J. Biol. Chem., 1913, 16.

(5) Porcher et Chevaluier, Le Lait, 1923, III. - Porcher, Bulletin de la Soc. de Chim. biol., 1, 923, V. 
Dans la seconde série d'expérience, j’ai eu comme collaborateur, pour l'extraction des gaz du lait, M. A.-P. Roller, et ces extractions ont été opérées dans le laboratoire du professeur HAckspils, de l'Institut de chimie de l'Université de Strasbourg.

II est d'observation courante que l'acidité du lait indiquée par le virage de la phénolphtaléine diminue sous l'influence du chauffage. De notre côté, nous avons constaté qu'il se produit également dans le lait soumis au vide une variation de cette réaction ne se traduisant pas toujours par une diminution.

Si au lieu de phénolphtaléine, dont la sensibilité ne porte que sur certaines acidités, on a recours aux indicateurs colorés ou au potentiomètre, on trouve, ainsi que nous l'avons constaté (1), que l'acidité globale ou " réelle » du lait indiquée par le $\mathrm{P}_{\mathrm{h}}$ ne subit aucun changement sensible par l'effet de la pasteurisation, pas plus qu'après extraction des gaz par le vide. Il semble donc qu'il se produise dans le lait aussitôt après le départ de l'acide carbonique, provoqué, soit par le vide, soit par le chauffage, un nouvel équilibre chimique que seule la phénolphtaléine nous signale, tandis qu'au contraire la mesure du $P_{h}$ ne nous en donne aucune indication précise. En conséquence, le simple titrage de l'acidité avec la phénolphtaléine comme indicateur nous a servi de guide dans le présent travail.

Nos observations ont porté sur plus de 50 extractions des gaz du lait et sur un nombre encore plus grand d'opérations de pasteurisation. Cette dernière était réalisée au moyen des trois différents procédés dont il est parlé dans cette étude.

Les deux premiers procédés qui sont de pratique usuelle provoquent une baisse de l'acidité par rapport à celle du lait cru témoin. Cette chute varie dans le même sens que la perte en acide carbonique par suite du chauffage.

Le lait pasteurisé en couche mince donne par contre une baisse d'acidité beaucoup plus petite qui correspond à une perte en acide carbonique très faible ou nulle. La moyenne des résultats peut se traduire par les quelques chiffres suivants qui indiquent l'ordre de grandeur du phénomène :

\begin{tabular}{|c|c|c|c|c|}
\hline 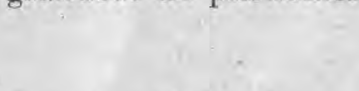 & $\begin{array}{c}\text { Lait } \\
\text { eru } \\
\text { témoin }\end{array}$ & $\begin{array}{l}\text { Lait soumis } \\
\text { a la pasteuri- } \\
\text { sation hante }\end{array}$ & $\begin{array}{l}\text { Lait soumis } \\
\text { a la pasteuri- } \\
\text { sation basse }\end{array}$ & $\begin{array}{l}\text { Lait soumis } \\
\text { à la pasteur. en } \\
\text { couche mince }\end{array}$ \\
\hline Teneur en $\mathrm{CO}^{3}$ ec. . & $4-3$ & 0,45 & $\overline{1.4}$ & $4-3$ \\
\hline Chute de l'acidité. .. . & ") & 0,9 & 0,7 & 0,3 \\
\hline
\end{tabular}

L'acidité est donnée en degrés SoxnLet qui expriment le nombre de centimètres cubes. de soude $\mathrm{N} / 4$ qu'il faut pour neutraliser $100 \mathrm{cc}$. de lait.

L'action du vide provoque le départ de tout l'acide carbonique

(1) Mlle Achand et H. Stassano. 
du lait, comme je l'ai signalé plus haut et démontré dans un travail précédent (1). Le lait présente alors en fin de l'opération une variation de l'acidité initiale particulièrement plus accentuée dans le cas du lait eru.

Cette variation est une baisse, la plupart du temps, mais peut devenir pendant la saison chaude une hausse. Le tableau qui suit donne les valeurs moyennes des variations d'acidité et montre que pour les laits pasteurisés la chute est d'autant plus accentuée qu'elle était faible de par l'effet de la température de pasteurisation.

\begin{tabular}{|c|c|c|c|c|}
\hline & $\begin{array}{l}\text { Lait } \\
\text { eru } \\
\text { témoin }\end{array}$ & $\begin{array}{c}\text { Lait } \\
\text { soumis a } \\
\text { la pasteur. } \\
\text { haute }\end{array}$ & $\begin{array}{c}\text { Lait } \\
\text { soumis a } \\
\text { la pasteur. } \\
\text { basse }\end{array}$ & $\begin{array}{l}\text { Lait soumis } \\
\text { à la pasteurisation } \\
\text { en couche mince }\end{array}$ \\
\hline
\end{tabular}

Nombre d'opérations. .

Moyenne des

variations d'acidité . . - $-0,5+0,1+0,5-0,2-0,35-0,70,0+3,2$

A plusieurs reprises, surtout pendant la saison chaude, le lait cru et aussi le lait chauffé en couche mince durant quelques secondes qui, par le fait de la très faible durée du chauffage, se trouve être celui des trois laits pasteurisés que la chaleur a le moins modifié, ont montré après le départ, causé par le vide, de l'acide carbonique, une tendance nette vers une augmentation de l'acidité. Les mêmes laits pasteurisés par les procédés courants ont, par contre, toujours montré une baisse.

Un fait analogue se produit avec la crème à $30 \%$. L'action du vide a donné sur l'échantillon cru une hausse d'acidité de 008 Soxhlet, tandis qu'elle a provoqué sur l'échantillon chauffé au bain-marie pendant une demi-heure à $80^{\circ}$ une chute de 0,7 .

L'interprétation des faits m'a conduit à considérer le lait comme un milieu minéral et organique des plus compliqués. Alors que le sang peut être envisagé comme étant surtout un tampon de carbonates et l'urine un tampon de phosphates, le lait semblerait être à la fois tampon de carbonates et de phosphates, sans compter encore les tampons correspondant aux sels des différents acides faibles existant en outre dans le lait (caséine, acide lactique, acide citrique, acides aminés).

Le départ de l'acide carbonique, pour quelque cause que ce soit, provoque une modification chimique du milieu qui tend à équilibrer la valeur du $\mathrm{P}_{\mathrm{h}}$, lequel reste autour des mêmes valeurs malgré la perte en acide carbonique. Dans les laits crus et dans les laits pasteu-

(1) C. R. de l'Académie des Sciences, V, CLXXIX, p. 438, 1924. 
risés en couche mince, c'est-à-dire les laits chauffés qui se rappróchent le plus des laits crus, l'effet tampon paraît plus accentué que dans les autres laits pasteurisés, les laits précisément traités par les procédés courants. Le départ complet de l'acide earbonique s'accompagne dans le lait cru d'une chute de l'acidité sensible à la phénolphtaléine qui oscille autour de 0,5 Soxhret, quelquefois même, nous l'avons signalé, il y a hausse ; il arrive de même pour les laits pasteurisés en couche mince. Pour les laits soumis à la pasteurisation haute et basse les chutes totales de l'acidité sont respectivement de 1,1 $(0,9+0,2)$ et de $1,05(0,7+0,35)$.

Les différents tampons se montrent done sensiblement retardés par les procédés usuels. Néanmoins, aussi dans les laits pasteurisés en couche mince, cet effet inhibiteur commence à se montrer lorsqu'on les porte à une plus haute température, à $90^{\circ}$ au lieu de $75^{\circ}$ et le même indice d'affaiblissement apparaît lorsque ces mêmes laits sont gardés pendant quelques jours à basse température. Il est vraisemblable, partant, que pareille modification de l'effet tampon relève d'une modification affectant particulièrement les constituants protéiques du lait les plus sensibles à la chaleur.

De ces deux séries de déterminations, il est à retenir en premier lieu : que le lait qui se rapproche le plus du lait $\mathrm{cru}$, à l'égard des constantes physico-chimiques, est le lait de pasteurisation en couche mince; en second lieu, il résulte de la deuxième série de déterminations : que l'effet tampon - lequel se produit dans le lait cru par suite du vide ou du chauffage - persiste presque intact dans le même lait de pasteurisation en couche mince, tandis qu'il ne se retrouve que fortement atténué, sinon modifié, dans les autres laits pasteurisés. Comme l'effet tampon en question est la conséquence de l'état normal des constituants du lait, particulièrement de ses constituants protéiques, la persistance plus ou moins complète de ce même effet tampon dans le lait de pasteurisation en couche mince est l'indice le plus sensible, le plus délicat de l'infiniment meilleure conservation du lait à la suite du chauflage d'après ce procédé de la couche mince. Il constitue donc l'un des points de repères strictement objectifs, parfaitement contrôlables, et qui permettront de distinguer nettement un lait de pasteurisation usuelle du lait traité par mon procédé.

La physiologie d'une part, par ses incessantes acquisitions dans le domaine de l'expérience, a contribué considérablement dans ces dernières années à faire apprécier la haute valeur nutritive du lait ; de l'autre côté, l'inaptitude grandissante chez la femme moderne des classes aisées, particulièrement, à l'allaitement au sein, a fait 
sentir tous les jours davantage l'utilité du lait dans l'alimentation. Désormais, à ce double titre, d'aliment de très grande valeur et de suppléant le lait maternel, le lait doit être envisagé à la fois comme la plus riche et la plus noble denrée.

On comprend que les Pouvoirs publics suivent avec intérêt les efforts individuels que l'on fait de tous eôtés pour augmenter et améliorer la production du lait. Il serait même à désirer qu'ils secondent ces efforts avec plus d'esprit de suite, qu'ils en encouragent même d'autres dirigés vers le même but par une législation appropriée et surtout en protégeant les producteurs consciencieux par le contrôle scientifique direct de la précieuse denrée.

De plus, les Pouvoirs publics et, dans l'espèce, les Gouvernements devraient prendre l'initiative d'appeler les savants des pays respectifs, les plus qualifiés par leurs études antérieures, à s'occuper d'une façon toute particulière du lait, ear il reste bien des points à éclaircir, bien des questions du plus haut intérêt pratique et social à résoudre à l'égard de cet aliment. Il y a, certes, beaucoup de savants tout indiqués pour s'occuper de cette étude, mais très peu d'eux s'en occupent de leur propre initiative. Il faudrait, en outre, leur en fournir les moyens.

En tout cas, les municipalités de plusieurs grandes villes d'Europe et d'Amérique, ont déjà décidé d'intervenir d'une fạcon plus ou moins directe dans tout ce qui concerne le lait. Quelques-unes ont été jusqu'à prendre l'initiative de la création de vacheries hygiéniques, mais la plus grande partie de ces municipalités se sont limitées à réglementer le ramassage à la campagne, les moyens de conservation et le transport du lait jusqu'à sa distribution dans les centres urbains. La municipalité de Milan, désireuse de suivre ce progrès, envoya l'année dernière plusieurs de ses conseillers avec l'adjoint au maire, visiter les grandes laiteries de Stockholm, Copenhague, Berlin et Strasbourg, qui, si elles ne sont entièrement des établissements municipaux, relèvent plus ou moins de l'Administration communale. Du rapport, rédigé par cette commission et dont le Corriere della Sera publia un long et circonstancié résumé, je me plais à retenir le renseignement suivant : Dans toutes ces grandes laiteries, le service de la distribution du lait pasteurisé en bouteilles bien cachetées, offrant des garanties sérieuses de propreté et d'hygiène, prend tous les jours une plus grande extension. On ne comprendrait pas autrement que ces laiteries, après s'être donné beaucoup de peine pour faire ramasser le lait à la campagne dans les meilleures conditions, notamment aussitôt trait, pour le faire pasteuriser le mieux qu'elles ont pu, c'est-à-dire en lui faisant perdre le moins de sa valeur alimentaire, se désintéresseraient à la fin complètement du sort qui est fatalement réservé au lait lorsqu'on le reçoit à la 
maison sans aucun souci d'hygiène. On commence alors par le faire bouillir dans une casserolle et voilà ainsi d'un coup détruit tous les efforts accomplis jusque-là dans l'espoir de fournir un lait sain et nourrissant. La distribution en bouteilles, dans des bouteilles absolument exemptes de microbes pathogènes, sinon tout à fait stériles, réalisée jusqu'au capsulage par des moyens mécaniques et à l'abri de contaminations dangereuses, s'impose désormais dans tous les centres où l'on veut effectuer l'allaitement artificiel des enfants ou l'alimentation exclusive des malades avec du lait convenablement pasteurisé.

Or, une organisation semblable ne peut pas être réalisée par un simple industriel, d'où l'avantage considérable de ces Administrations à fond municipal, mais régies par des eritériums industriels et commerciaux où l'intérêt général cependant n'est pas noyé par l'âpreté au gain dont difficilement parviennent à se libérer les entreprises privées. La Laiterie Centrale de Strasbourg représente précisément en France une organisation semblable, où l'esprit de progrès s'allie parfaitement aux principes d'économie les plus appropriés à une bonne administration. Et c'est grâce à cette excellente organisation, qui peut servir de modèle, que je dois de voir mon procédé sortir du domaine du laboratoire pour entrer dans la pratique. Et elle ira encore plus loin pour que les enfants et les malades puissent profiter de l'avantage principal de ce procédé, qui consiste à conserver le mieux et le plus eomplètement les constituants du lait, en mettant aseptiquement le lait à la sortie même du pasteurisateur en couche mince, dans des bouteilles rigoureusement aseptisées par des rincages successifs appropriés. Ces bouteilles seront ensuite capsulées avec des soins de même nature et aussi méticuleux, réalisés également par des moyens méeaniques.

Dans ces conditions, la laiterie pourra garantir la pureté du produit et dans les familles on n'aura qu'à chauffer simplement le lait à la température qui convient à son administration et celà seul au moment du besoin.

La Laiterie Centrale de Strasbourg se propose d'effectuer de la même manière le remplissage des fioles-biberon. Les mères n'auront qu'à tiédir le contenu au moment de la tétée et leurs enfants bénéficieront de ce fait d'un lait absolument sain et qui, à l'avantage de la cuisson modérée, dont il a été question plus haut, réunira celui de garder à l'état soluble les sels de chaux et les phosphates.

\section{CONCLUSION}

Pour ne pas dépasser outre mesure les limites de ce mémoire, la complexité du sujet, que je m'étais proposé d'y traiter, m'a obligé à effleurer à peine maintes questions qui méritaiènt d'être 
développées et de passer même sous silence bien d'autres questions, telles par exemple :

$1^{0}$ Le nettoyage du lait par fine tamisation convient bien mieux que l'essorage centrifuge qui aère le lait, lui enlève presque $10 \mathrm{ce}$. d'acide carbonique par litre et ne le débarrasse que de ces quelques milligrammes de terre qu'il tient en suspension par litre : ce que je peux affirmer d'après l'analyse chimique, bactériologique et cytologique des boues recueillies sur les parois des essoreuses;

$2^{\circ}$ L'avantage d'opérer dans les laiteries le déplacement de grandes masses de lait par la pression de l'acide carbonique, comme on opère dans les brasseries, au lieu que par les pompes de n'importe quel type ;

$3^{\circ}$ L'indication de transporter le lait des grands centres très éloignés de ramassage dans des vagons-citernes parfaitement stérilisables et infiniment plus faciles à remplir ou à vider aseptiquement que les pots laitiers et encore plus appropriés que ces derniers pour maintenir pendant le transport à la ville la température fraîche du lait sortant du réfrigérant après la pasteurisation; dans les lieux de destination; il serait très aisé de distribuer le lait ainsi convoyé dans les bouteilles selon les règles de l'hygiène.

En résumé, il y a à retenir, je crois, de mon long exposé, en premier lieu, l'utilité du lait modérément euit dans l'alimentation, particulièrement dans l'allaitement artificiel des enfants; en deuxième lieu, la nécessité de préserver dans le lait cuit le mieux qu'il soit possible tous les caractères du lait cru. Quelques-uns de ces caractères n'ont d'intérêt que comme indices, comme points de repère de la conservation des propriétés les plus fragiles et à la fois les plus nécessaires du lait. La présence dans les laits chauffés de ces propriétés n'est pas décelable directement ou immédiatement; et, enfin, en troisième lieu, la possibilité d'atteindre les deux buts, que l'on pouvait croire antinomiques, à savoir : l'assainissement parfait et constant du lait et la conservation presque complète de ses constituants, avec en plus les avantages de la cuisson modérée, par un procédé de pasteurisation caractérisé précisément par son maximum d'efficacité sur les microbes, combiné avec le minimum d'altération, dans les éléments de toutes sortes, du liquide qui les renferme, le lait dans notre cas.

Dans le rapport du directeur de la Laiterie Centrale de Strasbourg, que j'ai reproduit dans le chapitre IV de ce mémoire, on peut apprendre que le procédé de pasteurisation en question réunit, aux avantages d'ordre hygiénique exposés dans ces pages, bien d'autres avantages d'ordre économique.

Strasbourg, le 12 mai 1926. 